\title{
Novel Hypertension Management Model of Care Improves Blood Pressure Control in a West African Population
}

Isaac Kofi Owusu', Fred Adomako-Boateng ${ }^{2}$, Fred Kueffer ${ }^{3}$, Molly Guy ${ }^{3}$, Chemuttaai Lang'at ${ }^{3}$, Daniel B Grossman ${ }^{4}$, Teri Whitman ${ }^{3}$, Keith Holloman $^{3}$, Douglas A Hettrick ${ }^{3 *}$ and Opare-Sem $\mathbf{O}^{1}$

${ }^{1}$ Department of Medicine, School of Medical Sciences, College of Health Sciences, Kwame Nkrumah University of Science and Technology, Kumasi, Ghana ${ }^{2}$ Regional Health Directorate, Ghana Health Service, Kumasi, Ghana

${ }^{3}$ Medtronic, Inc, Minneapolis, MN, USA

${ }^{4}$ Mayo Clinic, Rochester, MN, USA

\section{Abstract}

Background: We developed and tested a novel hypertension management model of care designed for the resourcelimited setting of Sub-Saharan Africa.

Methods: The model consisted of a mobile tablet, automated BP machine, and a novel software application for longitudinal patient management. Patients were provided with a personalized management plan and attended regular $\mathrm{BP}$ local assessments and received real-time automated feedback. Clinicians remotely reviewed patient data, provided direct patient feedback and wrote electronic prescriptions directly to pharmacies.

Results: A cohort of 150 hypertensive patients ( $57 \pm 8$ years; $73 \%$ female) were enrolled. Average baseline BP was $135 \pm 18 / 84 \pm 10 \mathrm{mmHg}$ in the overall cohort and $153 \pm 13 / 90 \pm 11 \mathrm{mmHg}$ in the subgroup with uncontrolled hypertension $(n=58)$. After 6 months of recommended weekly BP monitoring, systolic blood pressure decreased significantly $(p<0.01)$ in both the overall cohort $(-4.7 \pm 18.7 \mathrm{mmHg})$ and in the uncontrolled subgroup $(-15.2 \pm 17.6 \mathrm{mmHg})$. The proportion of the population with uncontrolled hypertension decreased from $39 \%$ to $27 \%(p=0.01)$. Patient compliance with weekly BP assessments was $61 \%$ and 2,855 BP assessments were conducted.

In clinic patient visits were reduced $60 \%$ as compared to standard monthly visits. The software application directly referred 25 patients to a health facility (33 visits). Improvement in overall health awareness was reported in $82 \%$ of the participants and $95 \%$ of participants indicated a desire to continue using this model in the future.

Conclusions: Compliance and satisfaction with this multifaceted hypertension care model were high and led to significant and sustained decreases in blood pressure in this West African hypertensive population.

Keywords: Hypertension; Ghana; Model of care; Blood pressure control; Human-centered design

\section{Introduction}

Cardiovascular disease is currently the leading cause of death in sub-Saharan Africa in adults over the age of 30 [1]. The growing epidemic of uncontrolled hypertension (systolic blood pressure greater than $140 \mathrm{mmHg}$ ) remains the most important global contributor to cardiovascular morbidity and mortality leading to stroke, heart failure, kidney disease, and hypertensive emergencies [2,3]. By 2050 , hypertension prevalence is expected to expand to $33 \%$ of the population. The proportion of death and disability-adjusted life-years attributed to uncontrolled hypertension rose by an estimated $107 \%$ from 1990 to 2015 in West Africa [4]. In Ghana, nearly 30\% of the population has hypertension, but only $2.8 \%$ of those with hypertension are adequately controlled with pharmacologic management and lifestyle modifications [4]. This low rate of control is linked to multiple barriers to appropriate care, including high burden of travel to point of care, inequitable distribution of health facilities, inability for care providers to access longitudinal patient data, referral delays, and lack of patient awareness to the current state of their condition. Additionally, many hypertension patients are scheduled to visit the in-person clinic each month, regardless of the current clinical risk or status of their hypertension. When interviewed, several Ghanaian physicians noted that approximately two-thirds of the patients seen in hypertension clinics do not clinically require or benefit from monthly in-person medical reviews. Hence, these unnecessary visits create a substantial time and cost burden for the patient, and further congest an overcrowded system, preventing physicians from maximizing their time with those patients who require intense in-person assessment, review, and care. These barriers and challenges, coupled with rising rates of hypertension, require bold and innovative patient management solutions.

Medtronic Labs Empower Health ${ }^{\text {Tw }}$ (Medtronic Labs, Minneapolis, $\mathrm{MN}$ ) is a novel hypertension management model of care consisting of hardware and a proprietary software application. The application allows clinical users to collect basic clinical data, provide patients with earlier detection of elevated $\mathrm{BP}$, and remotely guide hypertensive patients to connect directly with their Health Care Provider (HCP) when further clinical evaluation is needed. HCPs can receive notifications on their mobile devices for patients needing evaluation, and may choose to communicate with patients on the application using Short Message Services (SMS). The application also allows HCPs to send electronic

*Corresponding author: Dr. Douglas A Hettrick, 710 Medtronic Parkway, Minneapolis, MN 55432, USA, Tel: +17636569053; E-mail: doug.hettrick@medtronic.com

Received November 14, 2018; Accepted November 26, 2018; Published December 04, 2018

Citation: Owusu IK, Adomako-Boateng F, Kueffer F, Guy M, Lang'at C, et al. (2018) Novel Hypertension Management Model of Care Improves Blood Pressure Control in a West African Population. J Hypertens (Los Angel) 7: 257. doi: 10.4172/21671095.1000257

Copyright: (C) 2018 Owusu IK, et al. This is an open-access article distributed under the terms of the Creative Commons Attribution License, which permits unrestricted use, distribution, and reproduction in any medium, provided the original author and source are credited. 
prescriptions directly to participating pharmacies. The Empower Health model of care was developed using human-centered design methodology, which focuses on co-creation of innovative solutions incorporating input from physicians, pharmacists, nurses, patients and their caregivers [5]. The model consisted of 4 major components including (1) generation of the patients' personalized follow-up plan, (2) community-based BP assessments (with associated patient messaging), (3) data triaging and HCP notifications, and (4) paperless (electronic) prescriptions for hypertension medications (Figure 1). The goal of Empower Health is to reduce the burden and improve the efficiency of managing hypertension for both patients and clinicians. The system was tested in a 6-month program called "Akoma Pa", (which means "healthy heart" in the local Twi language), enrolling patients previously diagnosed with hypertension.

\section{Methods}

The Empower Health system was tested in a 6-month program during which patients with a history of hypertension were enrolled using a convenience sampling method from Suntreso Government Hospital in Kumasi, Ghana. Ethical approval was obtained from the Committee for Human Research, Ethics and Publication at the School of Medical Sciences, KNUST, Kumasi, Ghana. Potential participants were evaluated for inclusion and exclusion criteria through a review of their medical records and a by routine in-person medical review. Primary inclusion criteria included patients 18-80 years of age with a diagnosis of hypertension with or without concomitant co-morbidities and a willingness to participate in all aspects of the protocol, including weekly BP assessments at a local pharmacy.

Prior to enrollment, evaluation for cardiovascular risks via laboratory investigations or review of medical charts was carried out for all participants. No new or additional clinical testing was required. Available clinic blood pressures for each subject were retrospectively retrieved from the patient record for post hoc comparison. Throughout the 6-month follow up period, patients continued to utilize their personal payment mechanisms for in-clinic medical reviews and medications (i.e. National Health Insurance, private insurance, or out of pocket payments).

Small reimbursements were provided to patients (a total of $\$ 15$ USD) to assist with transportation to in-clinic visits. Written informed consent was provided by each patient prior to enrollment.

\section{Enrollment and follow-up plan generation}

Clinical staff entered each patient's demographic and medical history data, as well as their baseline BP into the software application. Each patient's follow-up plan was created by the application's algorithms, which operationalize international guidelines [6,7] and enabled clinical risk categorization of patients based on comorbidities and risk factors. The follow-up plan was customizable at the health care provider's discretion for frequency of BP check assessments and inperson medical reviews.

Patients received training on the Akoma Pa program, including the individualized risk-based follow-up plan and their community-based BP check location options.

\section{Community-based BP measurements}

Each patient was asked to have his or her BP checked each week for the 6-month duration of the project. Patients could attend their BP assessment visits at any of the six geographically dispersed Akoma Pa community-based pharmacies, where staff had been trained to conduct the assessment visits. During each BP assessment visit, the patient provided the pharmacy trained staff with the reason for their visit, selfreported hypertension medication adherence, and current and recent physical symptoms. Three automated blood pressure measurements were then performed and recorded manually into the application by the trained staff. Based on the patient's enrollment risk classification, as well as their reported symptoms and blood pressure from that day, the patient received an application-generated personalized message. Depending on the patient's literacy levels, the message was either read by the patient or delivered verbally by the trained staff. The patient message included actionable information including positive verbal feedback, prompts to repeat blood pressure readings, or instructions to proceed immediately to the clinic or hospital for additional evaluation. Patients were not provided with home blood pressure monitors or any other technology and home monitoring was not required.

\section{Data triaging and HCP notifications}

When a patient received a message for referral following a community based BP check, the physician (or HCP assigned to receive the alerts) received an SMS message that a patient had been referred. The application was also available to the physician at any time,
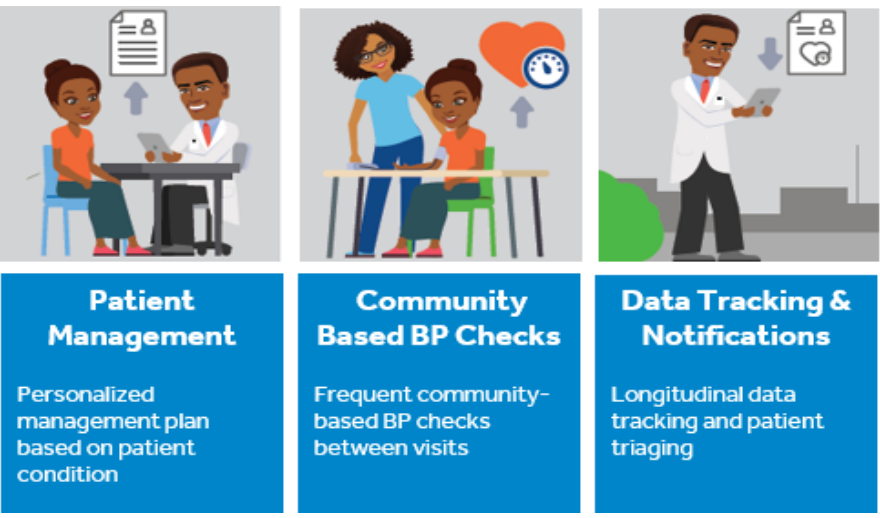

Notifications

Longitudinal data

tracking and patient triaging

Figure 1: Hypertension measurement model of care: Enrolled patients met initially with primary physician to determine a treatment plan. Each patient agreed to periodically visit a local pharmacy or facility for manual blood pressure checks by trained non clinical personnel. Recorded blood pressures were uploaded into the proprietary application via a tablet device which provided automated feedback directly to the patient. Managing physicians could use a smart device to remotely track serial blood pressure measurements, transmit prescriptions electronically to the local pharmacy and transmit messages directly to patients via SMS. Patients did not receive home monitoring equipment and no personal devices were required to participate. 
including during in-person visits, to review the patient's longitudinal data and follow-up on patients who had missed BP checks or medical reviews. The physician could send an SMS message to patients owning smart devices at any time via the application, communicating patientspecific follow-up instructions.

\section{Electronic prescriptions}

The software application provided a paperless mechanism for the physician to write hypertension-related prescriptions, selecting from hypertension-related medications listed in the Ghana Standard Treatment Guidelines (GSTG) [8]. Patients were then informed by SMS via the application that their prescription was available to be picked up at the participating pharmacy.

Commercially available BP monitors (Omron, Kyoto, Japan) were used for blood pressure measurements and the Empower Health software application was deployed on computer tablets (Samsung Galaxy Tab A). The primary physicians received the applicationgenerated patient notifications, via their personal smartphones. Inclinic medical review visits were conducted over the 6-month duration of the follow up, either every 30,60 , or 90 days. The frequency of these in-clinic medical reviews was determined by the risk classification performed by the Empower Health system algorithm at enrollment and confirmed or modified by the physician as clinically indicated. During those in-clinic medical reviews, the patient's provider had access to the longitudinal data collected during the weekly BP assessments. The provider could also make any updates to the $\mathrm{BP}$ assessment or in-clinic visit frequencies and/or update and prescribe hypertension-related medications via the application. During the in-clinic visits, the patients completed a medical review questionnaire and were asked questions regarding any recent clinic or hospital visits. At the final 6-month visit, patients completed a field tested and validated questionnaire that included questions regarding their personal acceptance of the system, their perception of the value of the Empower Health system, and any difficulties experienced using the system during the 6-months. Additional clinic blood pressure values for the 6-months prior to baseline were collected post hoc from the patients' medical record. The proportion of blood pressure readings below target was compared between the 6 months immediately prior to vs following enrollment. This proportion has previously been shown to be inversely related to cardiovascular risk $[9,10]$.

During the initial safety review, two independent Ghanaian cardiologist adjudicators ensured that the enrollment algorithm and the notification tier algorithm used during the $\mathrm{BP}$ assessment visits were processing the data correctly and were clinically accurate. A final adjudication assessed the possible relationship between reported cardiovascular events to any component or aspect of the Empower Health system.

\section{Statistical analysis}

Enrollment of 150 subjects afforded $86 \%$ power to test for a reduction in systolic blood pressure (SBP) of $5 \mathrm{mmHg}$ across the entire population (assumed standard deviation of $20 \mathrm{mmHg}$ ). Unless otherwise specified, analyses were performed using descriptive statistics including mean, standard deviation, median, minimum, and maximum for continuous variables and count and percentage for categorical variables. A t-test was utilized to determine if observed changes in blood pressure were different from zero. McNemar's test was utilized to determine if the proportion of subjects with uncontrolled hypertension changed from baseline to 6-months. SAS Software Versions 9.4 was used to conduct data processing and statistical analysis.

\section{Results}

A total of 150 patients from the Suntreso Hospital's Hypertension Clinic were enrolled between May 19, 2016 and June 21, 2016. Patients were recruited from 18 different neighbourhoods within the Kumasi area, the second largest metropolitan area in Ghana with approximately 1.7 million people. Average age was $57 \pm 8$ years; $73 \%$ of patients enrolled were female; $61 \%$ of patients reported either no schooling, primary school incomplete, or primary school complete as their highest level of education $36 \%$ reported no weekly income. Among wage earners, the average weekly income was 114 Ghana cedi (the equivalent of $\$ 25$ USD/week, which is slightly below that average Ghana 2016 GDP per capita of \$1,513.50 USD), with a range of 10 cedi/week- 425 cedi/week. The average baseline blood pressure was $135 / 84 \mathrm{mmHg}$. The enrolled cohort included 39\% with uncontrolled hypertension (SBP $\geq 140$ $\mathrm{mmHg}$ ). Patients were prescribed an average of $2.0 \pm 0.8$ hypertensive medications, including $88 \%$ on calcium channel blocker, $63 \%$ on ACE or $\mathrm{ARB}$, and $31 \%$ on thiazide diuretic.

The average travel time for each patient to the pharmacy by vehicle or bus was $20 \pm 14$ minutes. Patients attended BP assessment visits during all operating hours of the pharmacies, including early mornings, late evenings, and weekends. Compliance to completing the program was $100 \%$; no patients withdrew early, and all 150 patients completed a 6-month exit visit. A total of 363 medical reviews were completed in the 150 enrolled patients, as compared to the current standard of care that would have required a total of 900 medical review visits during the same duration of time with standard monthly visits (60\% reduction). Of the 150 patients, 92\% (138) completed all scheduled medical reviews as indicated by the software algorithm. A total of 2,705 blood pressure checks were performed following the enrollment visit through 6-months ( 0.71 checks/patient/week). Patient compliance with weekly blood pressure assessments was $61 \%$ (Figure 2). The rate of compliance decreased during the initial 2-3 months of follow up, but then plateaued at more than $55 \%$ per week in the final months. Throughout the 6-months, 654 SMS messages were sent from the physicians directly to the patients' personal smart device, through the software application. Each patient was sent at least 2 messages, with an average of $4.4 \pm 1.4$ messages sent per subject.

The software application algorithm logic directly referred $17 \%$ of the patients to a health facility following a community-based $\mathrm{BP}$ assessment (25 patients during 33 visits, representing $1 \%$ of the

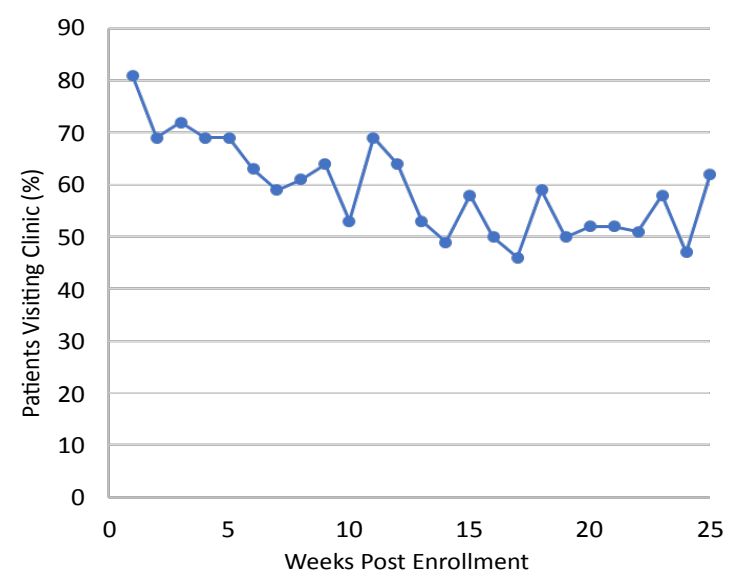

Figure 2a: Overall population compliance remained over $50 \%$ throughout the 6-month study period. 


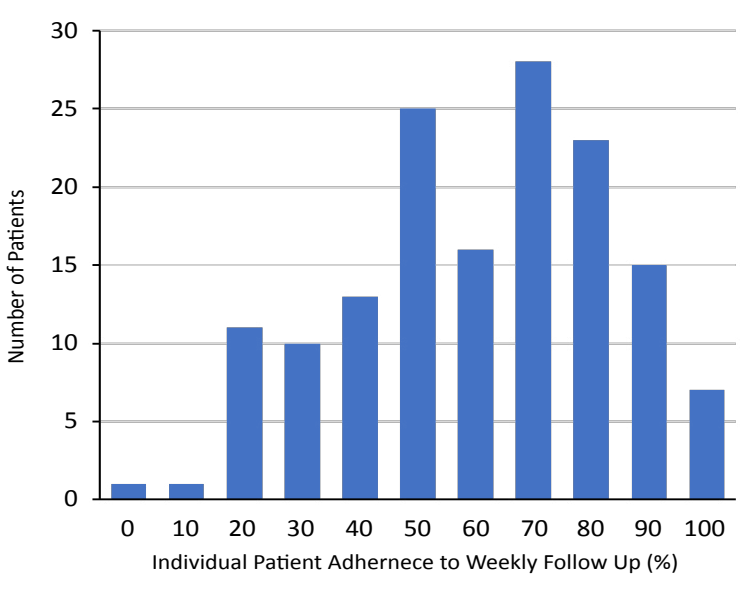

Figure 2b: Histogram of individual patient in clinic blood pressure check compliance: Most patients attended $60 \%$ or more of required weekly BP checks.

total community-based BP assessments). No deaths occurred, and 6 patients (4\%) required inpatient hospitalization. Five out of the six hospitalizations were related to cardiovascular conditions as reported by patient recall (i.e. high blood pressure, swelling of the tongue/lips and reported fatigue, hypotension, difficulty breathing, and stroke). Of those, 3 hospitalizations were initiated by the Empower Health system following a BP assessment at a pharmacy and 2 hospitalizations were initiated by the patient on non-BP assessment days, which included one stroke. Due to non-availability of the patient's prescribed antihypertensive medications at the time of the event, the stroke hospitalization was adjudicated as unrelated to the Empower Health system. No events or hospitalizations were adjudicated as being related to the Empower Health system.

Improvement in overall health awareness was reported in $82 \%$ of the participants, and $95 \%$ of participants indicated a desire to continue using the program in the future. Likewise, the physicians indicated satisfaction with the model of care and the positive impact it had on their clinic workflow, specifically the $60 \%$ reduction in non-medically required clinic appointments and the ability to write prescriptions electronically.

Average BP was $135 / 84 \pm 18 / 10 \mathrm{mmHg}$ in the overall cohort at baseline, and 137/85 $\pm 14 / 8$ during the full 6 -month period prior to enrollment (average $3.5 \pm 1.1$, measurements per patient) (Table 1).

Likewise, the pre-enrollment 6-month average blood pressure was $151 / 90 \pm 11 / 7$ in the subgroup with uncontrolled hypertension defined as average pre-enrolment blood pressure $\geq 140 \mathrm{mmHg} \quad(\mathrm{n}=55)$, and $128 / 82 \pm 7 / 7$ in the subgroup with controlled blood pressure during the prior 6 months defined as average systolic blood pressure $<140 \mathrm{mmHg}$ ) $(\mathrm{n}=83)$ (Table 2). During the 6 months of voluntary weekly monitoring, average systolic blood pressure decreased significantly in the overall cohort $(-6.2 \pm 12.6 \mathrm{mmHg} ; \mathrm{p}<0.001)$ and in the uncontrolled subgroup $(-13.1 \pm 12.9 \mathrm{mmHg} ; \mathrm{p}<0.001)$, while systolic blood pressure remained constant in the sub group with controlled pressure at baseline $(-1.6 \pm$ $10.1 \mathrm{mmHg}$; $=0.16$ ) (Figure 3). The proportion of "controlled" blood pressure readings defined as systolic blood pressure below $140 \mathrm{mmHg}$ increased significantly in the overall cohort as well as both sub groups, including the group with "controlled" blood pressure during the 6 months prior to enrolment (Figure 4).

\begin{tabular}{|c|c|}
\hline Age (years) & $57 \pm 8$ \\
\hline Gender (\% female) & $73 \%$ \\
\hline $\mathrm{BMI}\left(\mathrm{kg} / \mathrm{m}^{2}\right)$ & $29 \pm 5$ \\
\hline $\mathrm{SBP}(\mathrm{mmHg})$ & $135 \pm 18$ \\
\hline $\mathrm{DBP}(\mathrm{mmHg})$ & $84 \pm 10$ \\
\hline$\%$ with uncontrolled hypertension ( $\geq 140 \mathrm{SBP}$ ) & $39 \%$ \\
\hline SBP in uncontrolled patients & $153 \pm 13$ \\
\hline DBP in uncontrolled patients & $90 \pm 11$ \\
\hline Education (\% completing secondary school or higher) & $35 \%$ \\
\hline Years diagnosed with hypertension ${ }^{1}$ & $9 \pm 8$ \\
\hline Average \# of HTN meds per patient & $2.0 \pm 0.8$ \\
\hline Baseline Medication by Class ( $\%$ of patients): & \\
\hline ACE or ARB & $63 \%$ \\
\hline Aldosterone antagonist & $1 \%$ \\
\hline Beta blocker & $1 \%$ \\
\hline Calcium channel blocker & $88 \%$ \\
\hline Central-acting agent & $3 \%$ \\
\hline \multirow[t]{3}{*}{ Travel Time to Clinic } & $<30$ mins: $51 \%$ \\
\hline & $30-60$ mins: $39 \%$ \\
\hline & $1-2$ hours: $10 \%$ \\
\hline \multirow[t]{4}{*}{ Mode of transportation } & Personal vehicle: $5 \%$ \\
\hline & Taxi: $19 \%$ \\
\hline & Bus: $63 \%$ \\
\hline & Walked: $13 \%$ \\
\hline \multicolumn{2}{|l|}{ Comorbidities $^{2}$} \\
\hline None & $29 \%$ \\
\hline Family History of Cardiovascular Disease & $46 \%$ \\
\hline Obesity & $33 \%$ \\
\hline Diabetes & $25 \%$ \\
\hline History of Stroke & $4 \%$ \\
\hline \multirow{4}{*}{$\begin{array}{l}\text { Pregnancy } \\
\text { Was the patient ever diagnosed with pre-eclampsia or } \\
\text { high BP during any pregnancy ( } n=110 \text { females)? }\end{array}$} & NA: $1 \%$ \\
\hline & No: $80 \%$ \\
\hline & Yes: $18 \%$ \\
\hline & Unknown: $1 \%$ \\
\hline
\end{tabular}

Table 1: Baseline population demographics.

\begin{tabular}{|c|c|c|c|}
\hline & $\begin{array}{c}\text { All subjects } \\
(\mathbf{n}=\mathbf{1 3 8})\end{array}$ & $\begin{array}{c}\text { Uncontrolled } \\
(\mathbf{n}=\mathbf{5 5})\end{array}$ & $\begin{array}{c}\text { Controlled } \\
(\mathbf{n}=\mathbf{8 3})\end{array}$ \\
\hline $\begin{array}{c}\text { Pre Enrollment Average } \\
\text { SBP (mmH) }\end{array}$ & $137.4 \pm 14.0$ & $150.9 \pm 10.9$ & $128.4 \pm 6.5$ \\
\hline $\begin{array}{c}\text { Within Study Average SBP } \\
\text { (mmHg) }\end{array}$ & $131.2 \pm 12.7$ & $137.8 \pm 14.5$ & $126.8 \pm 9.1$ \\
\hline Difference (mmHg) & $-6.2 \pm 12.6$ & $-13.1 \pm 12.9$ & $-1.6 \pm 10.1$ \\
\hline p-value & $<0.001$ & $<0.001$ & 0.16 \\
\hline
\end{tabular}

Table 2: Error! Main document only. Change in blood pressure prior to and following.

\section{Discussion}

This prospective investigation demonstrated the utility of a novel model of community based healthcare to address significant barriers to the control of hypertension in a Western African population. Empower Health is a locally-appropriate novel hypertension management model of care that consists of commercially available hardware and a propriety software application.

The model was designed specifically for the unique needs of resource-limited settings using a human-centered design framework. This human centered design methodology focuses on understanding the unique unmet needs of the patients, local stakeholders, and health systems.

The co-created model of care integrated frequent community- 


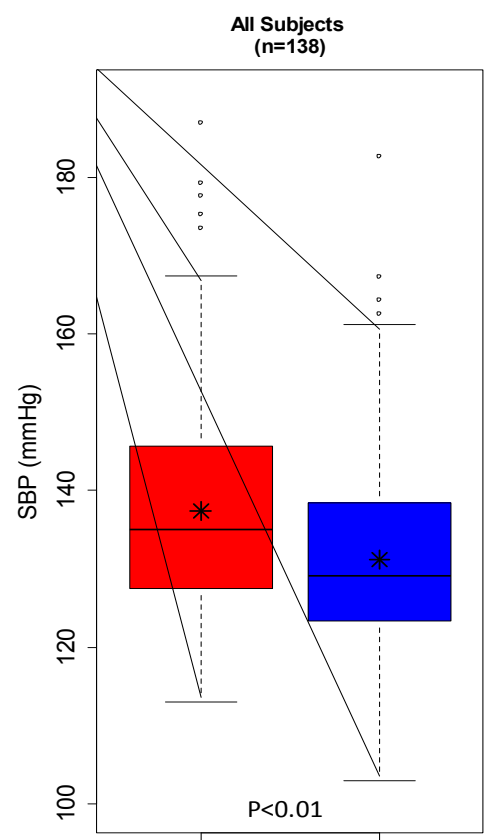

Pre-Enrollment Within Study

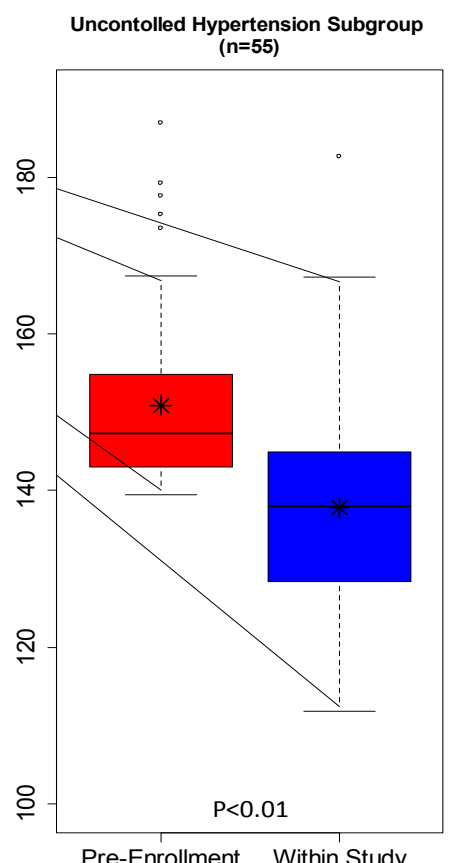

Pre-Enrollment Within Study

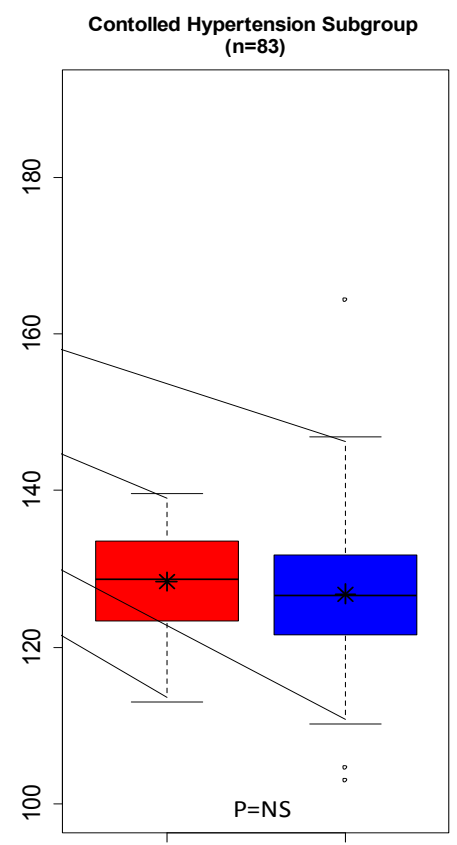

Pre-Enrollment Within Study

Figure 3: Changes in blood pressure during the 6 months prior to program enrollment obtained from the patient record (red) compared to average blood pressure during the 6 month follow up period (blue) for the all subjects with pre enrollment data available (left), the subset of patients with average systolic blood pressure $\geq 140 \mathrm{mmHg}$ during the 6-month pre-enrollment period ("uncontrolled" group, center), and the sub group with average systolic blood pressure $<140 \mathrm{mmHg}$ during the 6 months prior ("controlled" group, right): Blood pressure decreased significantly in the full cohort and the uncontrolled subgroup but not in the controlled sub group.
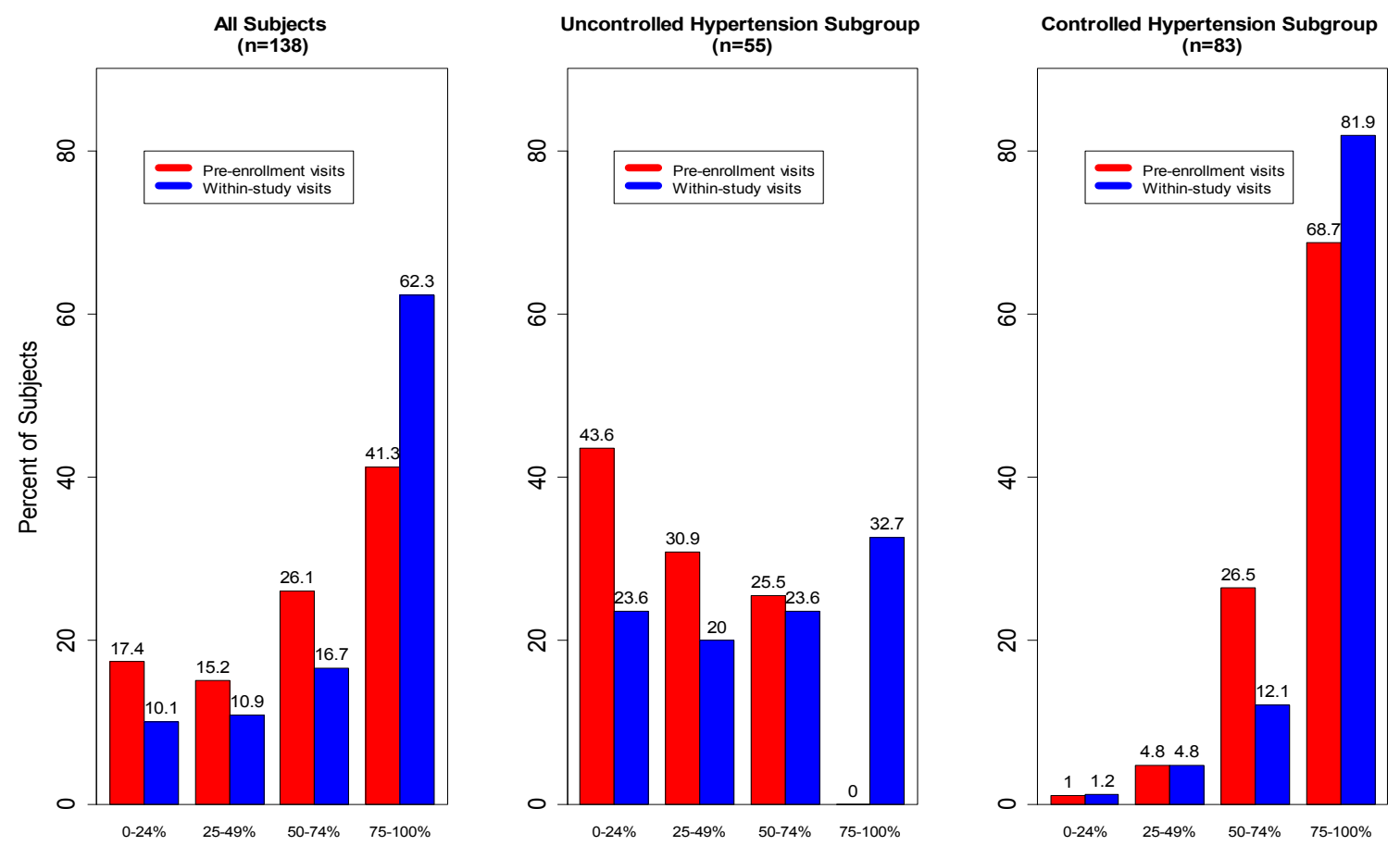

Percent of Visits with SBP $<140 \mathrm{mmHg}$

Figure 4: Proportion of blood pressure readings $<140 \mathrm{mmHg}$ during the 6-month pre enrollment period (red) compared to the 6-moth enrollment period (blue) for the entire cohort (left), the subset of patients with average systolic blood pressure $\geq 140 \mathrm{mmHg}$ during the 6 -month pre-enrollment period ("uncontrolled" group, center), and the sub group with average systolic blood pressure $<140 \mathrm{mmHg}$ during the 6 months prior ("controlled" group, right): The distribution shifts to the right significantly for all three groups including the sub group classified as having "controlled" blood pressure during the preenrollment period. 
based blood pressure assessments with patient-centered remote monitoring and allowed for increased active patient engagement, access to health information, and real-time analysis and feedback.

The Akoma Pa program resulted in blood pressure drops across the entire cohort, with the largest decreases in systolic blood pressure in the majority subset of patients with uncontrolled blood pressure prior to enrollment. No patients withdrew early and compliance to completing weekly BP assessments and scheduled in-person medical reviews was high throughout the 6-month follow up. Patients also expressed a very high level of satisfaction with the program and enthusiasm to continue with the program. The presently observed drops in blood pressure could have meaningful clinical impact, since similar drops have been associated with significant reductions in cardiovascular risk, including coronary heart disease events (by about $25 \%$ ), stroke (by about $41 \%$ ), and heart failure (by about 25\%) [11]. The observed reductions in blood pressure, combined with the observed $60 \%$ reduction in office visits for the cohort suggest that the present locally appropriate model of care may increase efficiency and reduce costs for patients and health systems. Such improvements may be particularly important in geographies where health facilities are overwhelmed by demand and access to healthcare, for patients, is difficult.

We also observed significant increases in the proportion of blood pressure readings less than $140 \mathrm{mmHg}$ in the full cohort as well as both sub groups including the group with controlled" blood pressure during the 6 months prior to enrollment. This proportion has been shown to be inversely proportional to cardiovascular risk $[9,10]$, implying that even patients with relatively well controlled hypertension may also experience reduced cardiovascular risk following enrollment in the program.

The present results have important implications for the hypertensive population beyond Ghana. The African population is forecasted to exceed 2.4 billion by the year 2050 , representing $25 \%$ of the global population [12] and hypertension remains a growing epidemic. Estimates of the prevalence of hypertension in Africa are among the world's highest at up to $46 \%$. Conversely, hypertension control rates in Africa are among the world's lowest at $6.5 \%$, explained in part by low hypertension awareness levels of just $34 \%[13,14]$.

Unlike the presently described Empower Health System, currently ongoing efforts to improve hypertension care in Africa include multiple screening programs aimed at improving the awareness of people living with hypertension, as well as programs focused on increasing the availability of hypertension-related medications $[15,16]$. Previously developed and marketed tools are designed to provide and document patient home BP readings. However, these systems lack clinical data analysis tied to triaging based on international standard guidelines, physician alert notifications, personalized SMS messaging to patients, and electronic prescriptions.

Additionally, the existing models of improved care are not designed to address barriers to care including linking patients into a care management system, maintaining patient and engagement and support, time and cost burden of travelling to the clinic, lack of patient awareness to the current state of their condition, and the lack of readily available longitudinal data for their physicians to make data-driven care decisions. The goal of the present model is to support the primary physician by creating a more efficient in-clinic experience for those patients who need greater in-person review time. The novel features of this model of care, created to specifically address critical locally observed barriers to hypertension treatment, likely contributed to both the high observed levels of patient compliance and the sustained decreases in systolic blood pressure.

The present analysis has limitations. No control arm was studied for comparison. However, we demonstrated gradual, consistent and sustained decreases in blood pressure within a population with previously well documented low rates of hypertension control. In addition, we compared the average blood pressure during the 6-month study period with average blood pressure measured during the 6 months immediately prior to enrollment obtained from the patient record.

In summary, a novel hypertension monitoring and remote management application focused on community-based assessments, early detection of warning signs, and algorithm-based remote monitoring resulted in decreased blood pressure, and the proportion of controlled blood pressure readings, that was sustained over six months in this West African population. Results were driven by strong patient compliance and satisfaction and increased access to community-based care. Because of the observed decreases in blood pressure, the model has potential for substantial economic health care savings for both patients, providers, and the health care systems in both private and public sectors. Based on these favorable results, the Empower Health model of care should be used by health systems in Ghana and additional geographies with similar barriers to access to care. Further research is warranted to understand the applicability of this model of care to other disease states and use cases that would benefit from increased patient awareness of their condition, remote monitoring options, and increased access to longitudinal data.

\section{Acknowledgments}

We would like to thank Dr. Alexis Nang-Beifubah, Regional Health Director of the Ashanti Region, and Dr. Akumia, Medical Director at Suntreso Hospital for their unwavering support throughout the project. We would also like to thank Dr. Lambert Appiah and Dr. Emmanuel Acheamfour-Akowuah for their clinical guidance and adjudication efforts. We are grateful to the Suntreso Hypertension Clinic staff for their dedication to the project and the patients. We would like to acknowledge ClickMedix for their partnership during the Akoma Pa program. And finally, we are forever thankful to the 150 patients who participated in this project, as well as the hundreds of individuals we interviewed over the last three years as we worked side by side to co- create this model of care.

\section{Funding}

The program was funded by Medtronic, Inc. Minneapolis, MN. USA.

\section{References}

1. Gaziano TA (2005) Cardiovascular disease in the developing world and its cost-effective management. Circulation 112: 3547-3553.

2. Peck RN, Green E, Mtabaji J, Majinge C, Smart LR, et al. (2013) Hypertensionrelated diseases as a common cause of hospital mortality in Tanzania: a 3-year prospective study. J Hypertens 31: 1806-1811.

3. Owusu M, Nguah SB, Boaitey YA, Badu-Boateng E, Abubakr AR, et al. (2012) Aetiological agents of cerebrospinal meningitis: a retrospective study from a teaching hospital in Ghana. Ann Clin Microbiol Antimicrob 11: 28.

4. Cappuccio FP, Micah FB, Emmett L, Kerry SM, Antwi S, et al. (2004 Prevalence, detection, management, and control of hypertension in Ashanti, West Africa. Hypertension 43: 1017-1022.

5. Matheson GO, Pacione C, Shultz RK, Klügl M (2015) Leveraging humancentered design in chronic disease prevention. Am J Prev Med 48: 472-479.

6. Mancia G, Fagard R, Narkiewicz K, Redon J, Zanchetti A, et al. (2013) 2013 ESH/ESC Guidelines for the management of arterial hypertension: the Task Force for the management of arterial hypertension of the European Society of Hypertension (ESH) and of the European Society of Cardiology (ESC). Eur Heart J 34: 2159-2219.

7. Chobanian AV, Bakris GL, Black HR, Cushman WC, Green LA, et al. (2003) The Seventh Report of the Joint National Committee on Prevention, Detection, 
Citation: Owusu IK, Adomako-Boateng F, Kueffer F, Guy M, Lang'at C, et al. (2018) Novel Hypertension Management Model of Care Improves Blood Pressure Control in a West African Population. J Hypertens (Los Angel) 7: 257. doi: 10.4172/2167-1095.1000257

Page 7 of 7

Evaluation, and Treatment of High Blood Pressure: the JNC 7 report. JAMA. 289: $2560-2572$

8. Standard Treatment Guidelines. Ministry of Health (GNDP) Ghana. Sixth Edition, 2010.

9. Mancia G, Schumacher H, Redon J, Verdecchia P, Schmieder R, et al (2011) Blood pressure targets recommended by guidelines and incidence of cardiovascular and renal events in the Ongoing Telmisartan Alone and in Combination With Ramipril Global Endpoint Trial (ONTARGET). Circulation 124: $1727-1736$.

10. Mancia G, Kjeldsen SE, Zappe DH, Holzhauer B, Hua TA, et al. (2016) Cardiovascular outcomes at different on-treatment blood pressures in the hypertensive patients of the VALUE trial. Eur Heart J 37: 955-964.

11. Law MR, Morris JK, Wald NJ (2009) Use of blood pressure lowering drugs in the prevention of cardiovascular disease: meta-analysis of 147 randomised trials in the context of expectations from prospective epidemiological studies. BMJ 338: b1665.

12. https://esa.un.org/unpd/wpp/publications/files/wpp2017_keyfindings.pdf

13. World Health Organization. Global status report on noncommunicable diseases. WHO, Geneva. 2010.

14. Chow CK, Teo KK, Rangarajan S, Islam S, Gupta R, et al. (2013) Prevalence, awareness, treatment, and control of hypertension in rural and urban communities in high-, middle-, and low-income countries. JAMA 310: 959-968.

15. Vedanthan R, Blank E, Tuikong N, Kamano J, Misoi L, et al. (2015) Usability and feasibility of a tablet-based Decision-Support and Integrated Recordkeeping (DESIRE) tool in the nurse management of hypertension in rural western Kenya. Int J Med Inform 84: 207-219.

16. Lamptey P, Laar A, Adler AJ, Dirks R, Caldwell A, et al. (2017) Evaluation of a community-based hypertension improvement program (ComHIP) in Ghana: data from a baseline survey. BMC Public Health 17: 368. 